\title{
La Filosofía, el Estado y LA REvolución*
}

\section{De la Filosofía fuera de sí a la Filosofía en común}

Mi generación de profesores de filosofia no ha vivido nunca en la evidencia su relación con el ejercicio de la reflexión ni con la práctica de la enseñanza. Dividida desde sus primeros pasos entre la ciencia y la rebelión, ha reivindicado siempre una impureza necesaria de la filosofia. Obstinadamente ha afirmado la relación desigual que ésta mantiene (incluso y sobre todo en su exigencia crítica) con lo que no es ella, singularmente con la política.

Estudiantes, nuestro horizonte había sido el del fin de la metafisica. Nuestros mayores nos habían hecho leer a Nietzsche, Marx y Freud, teniendo como perspectiva un mismo diagnóstico: en adelante es imposible pensar el ser como un principio único, a partir del cual todo lo que se manifiesta podría llegar a ser transparente e inteligible. Nuestros maestros también habia decretado que la revolución no estaba a la orden del día. Más valía ocuparse en constituir un stock de armás conceptuales que, llegado el momento, podrían servir.

Con Mayo de 1968, esta concepción de una autonomía de la filosofia en relación con la política iba a volar en pedazos. La cuestión dejó de ser: "¿para qué sirve la filosofia?", y se convirtió en: "¿a quién, entonces, sirve la filosofia?".

- Editado originalmente en: Itens y Vulumes, Ne 98/98, diciembre de 1995, Bogotá, Colombia. Su publicación en listudios Sociales fue autorizada por el autor.

** Centro de Estudios Políticos de la Sorbona, París, Francia. 
Entiéndase: "¿sirve al pueblo o a sus enemigos?". Pero con Canguilhem, Deleuze y Foucault pronto nos encontramos más allá del modelo sartriano del filósofo como iṇtelectual al servicio del pueblo. Lo que vino a sustituirlo fue la impugnación del saber mismo.

Proyectamos organizar la resistencia contra todo lo que calificaba institucionalmente ese saber, y que Jacques Rancière ha descrito en estos términos la posición de dominio, la división de los roles (en el cuerpo social: la élite y la masa ignorante; en la institución escolar y universitaria: el profesor, los alumnos), el reconocimiento académico y el control de la jerarquía, en fin, la certeza de lo verdadero. A partir de la crítica de la división entre trabajo manual e intelectual, pensamos devolver la palabra al pueblo. Esta liberación tomó la forma de una lucha contra todas las opresiones localizadas y por la supresión radical de todos los poderes, excepto el poder revolucionario.

¿Cómo el fin de este sueño de emancipación radical se ha transformado en el comienzo de una aventura del pensamiento? Entre los múltiples signos de que una cierta figura de la filosofia transformada en política se había derrumbado, citaré uno, ciertamente menor, pero que para mí no dejó de tener sus efectos: el fracaso, en 1974, del proyecto de la emisiones Sartre en la televisión, debido, sin duda, tanto al eclipse histórico de la imagen de la liquidación del viejo intelectual que debía callarse para dejar hablar a la voz del pueblo (cf. Rancière), como a los obstáculos levantados por el poder político para impedir esas emisiones. Se trataba de seguir a Sartre en el siglo, no como individuo singular sino como subjetividad abstracta: trazar el balance, de alguna manera, de las formas de la rebelión y de las metamorfosis de la noción de autoridad. Del grupo "revueltas obreras", que reunía, con Jean Borreil y Jacques Rancière, a algunos de los jóvenes filósofos del 68 agrupados alrededor de Sartre, y que pretendian extraer, de los archivos y de la memoria oral del pueblo, la materia de otro relato de la historia contemporánea de Francia, ha nacido el Centro de investigación sobre las ideologias de la rebelión y su revista: Las Rebeliones Lógicas.

Es en este marco en el que, con Stéphane Douailler, he intentado analizar la elaboración y la puesta en obra de las estrategias modernas de poder sobre el niño: la preservación de su cuerpo y la educación de su alma, que participan de ese movimiento general del siglo XIX que va de la filantropía privada a una política de asistencia administrada por las instituciones del Estado. Estudiando el trabajo de los niños y la ley del 22 de marzo de 1841, fuimos llevados a poner en causa la interpretación marxista clásica, según la cual toda ley social sería el efecto de un retroceso de la clase dominante y el objeto de una victoria del proletariado. $\mathrm{Y}$ pudimos describir la puesta en obra de una política de la fuerza de trabajo y de un proyecto de moralización de las clases pobres por el Estado liberal moderno, perceptible en toda la legislación del trabajo. ${ }^{(1)} \mathrm{A}$ partir de allí era posible mostrar, no solamente cómo la historia se orientaba hacia el reconocimiento de un derecho de inspección del Estado sobre los asuntos privados - aquellos de la fábrica como 
aquellos de familia - , sino también dar cuenta de proyectos positivos que manifiestan esta estrategia de control social. La cuestión no cra más la de la reglamentación del espacio privado, sino la de la construcción de un modelo global de educación susceptible de reemplazar a la familia y a la fábrica ante el niño, y de su experimentación sobre una fracción de la población interesada.

Paradójicamente, este modelo no ha sido, en primer lugar, el de la escolarización, sino el del trabajo agrícola en las primeras colonias penitenciarias, que resulta directamente de las teorias del confinamiento carcelario y de su impugnación en el seno mismo de las sociedades filantrópicas encargadas de su propagación y de las técnicas disciplinarias de reorientación moral puestas en obra en el medio carcelario. Hemos partido así de la toma en consideración, en la cuestión penitenciaria, de los niños moralmente desfavorecidos: o sea aquellos que el código penal francés define, en su arúculo 66, como debiendo ser absueltos por haber actuado sin discernimiento, para descubrir poco a poco que, en el traslado de este niño de la penitenciaría a la colonia agrícola - asunto de vivos debates entre 1830 y 1860 - se juega algo más que su suerte específica, a saber: la producción de una estrategia de moralización del pueblo que hace aparecer a la prisión, bajo la forma particular de la colonia penitenciaria, como portadora de la solución de la cuestión social. La historia del fracaso de la constitución de eso que Gustave Vapereau, profesor de filosofia y discipulo de Victor Cousin, llamaba una "familia artificial", (2) condujo a un fraccionamiento que divide la infancia según categorias heterogéneas, tomadas por su cuenta por instituciones especificas: los criminales, los tarados, los sin familia y los indigentes dependen cada uno de una política, de represión para los primeros, de cuidados médicos para los segundos y de asistencia para los otros. ${ }^{(3)}$ Es por este rodeo paradojal que he llegado al estudio de la instauración paralela del modo de educación específico destinado a los sujetos que se supone llegarán a ser los cuadros del Estado: o sea la constitución de la enseñanza filosófica para uso de aquellos que Victor Cousin denomina "la aristocracia legítima" de los colegios, que procede de una institucionalización de la filosofia, cuya genealogía se intenta restituir aquí.

Este orden de causalidad se doblaba en otra lógica. habíamos llegado a ser profesores y nos correspondía, a nuestro turno, enseñar la filosofia. En el momento en que empezábamos, ésta era el objeto de ataques organizados por la política y la ideología tecnocrática del poder liberal establecida como materia de enseñanza. Nos encontramos emprendiendo un vasto movimiento de defensa de nuestra disciplina. La defendimos, en primer lugar, porque era nuestra profesión. La defendimos también, y ardientemente, porque no servia para nada. ${ }^{(4)}$

Pensábamos, en efecto, que el Estado liberal quería reemplazar la filosofia por las ciencias humanas, la psicologia, la sociologia, y toda suerte de formaciones prácticas confiadas a técnicos de los saberes. La filosofía era "una pérdida de tiempo", según la expresión de un ministro de la época. En una palabra, no éramos rentables. Por consiguiente, luchamos por el maritenimiento oficial de nuestra 
inutilidad, y por la existencia en la enseñanza de este momento filosófico. Habíamos fundado una pequeña revista, Le Doctrinal de Sapience, seguida pronto por una efimera colección de libros, los Almanachs du Philosophe Boîteux, ${ }^{(5)}$ en los cuales objetamos las razones adelantadas por la institución filosófica para su autodefensa y buscamos una legitimación más conforme a nuestras convicciones contestatarias. Nuestros mayores se habían dividido interiormente: ellos separaban, en el tiempo y en el espacio, su oficio filosófico de su lucha política, y podían, adoptando un punto de vista materialista, explicar a Descartes en clase, sin cuestionar la forma institucional del ejercicio. Althusser nos había llamado al orden de la lucha de clases en la teoría, y su reivindicación de una autonomía de la filosofia contra la reducción de ésta a la política, iba a la par con una condena sin apelación del boicot a la agregación. Por lo que a nosotros respecta, pretendimos inventar una relación más auténtica entre las lecciones extraídas de nuestro reciente pasado contestatario y nuestra práctica actual de la enseñanza filosófica. Pero, ¿cómo conciliar esta exigencia crítica con la defensa de la institución? Segunda figura de la paradoja de nuestra generación: los hijos de Nietzsche, de Marx y de Freud se convertían en los defensores de la filosofia escolar y universitaria. La generación que siguió inmediatamente a la nuestra había fundado una revista que tenía por título: Oui la Philosophie!

Es esto lo que me ha llevado también a trabajar sobre la genealogía de la institución filosófica, y a remontarme a ese momento constitutivo - los años 1830 - en que la filosofía se convirtió en un asunto de enseñantes retribuidos por el Estado. Especialmente me he interrogado sobre la distancia que podía ahondarse entre el ideal del filósofo y la realidad de su enseñanza, ${ }^{(6)}$ cuestión que desembocaba en aquella de los criterios de aceptación del discurso filosófico en las instituciones. ${ }^{(7)}$

¿Qué es, por ejemplo, lo que permite decidir que tal frase pueda ser pronunciada en una cátedra de filosofia en la Universidad, y que tal otra no deba serlo? ¿Cómo separar lo que es propiamente filosófico de lo que no lo es, o se supone no serlo: el periodismo, la literatura, la política? Igualmente, yo estudiaba cómo se constituye, en el siglo XIX, la idea de una filosofia reservada a aquellos que tienen el ocio y la capacidad de acceder a la verdad, mientras que, por incapacidad constitutiva o por falta de ocio, la gran mayoria estaría condenada a la creencia: "es necesaria una religión para el pueblo". Sobre este terreno baldío, mis interrogaciones reencontraban las que Miguel Abensour había formulado en sus trabajos sobre Pierre Leroux, ${ }^{(8)}$ una de las figuras más radicales de la impugnación de la institución filosófica. Más aún, él me incitaba a buscar, del lado de la ciencia política, los instrumentos conceptuales que yo necesitaba para analizar la política de la filosofia en el orden de sus razones agonísticas, y no solamente en el de sus filiaciones conceptuales. Centrando mi trabajo sobre las crisis internas y externas a la institución, he podido descubrir que el espiritualismo universitario francés participaba 
directamente del paradigma del naciente Estado liberal moderno, y he podido comprometerme en el proyecto de emprender una historia política de la filosofia.

Quisiera señalar, antes de describir los requisitos de esa historia, cómo ese rodeo por el siglo XIX y ese recurso a la ciencia política se inscriben en las cuestiones de hoy. Para mi generación - se lo habrá comprendido-, defender la filosofia no era volver a un orden antiguo, sino participar en una lucha por la democracia en el pensamiento, por la comunidad de los espíritus. Los Etats Gènèraux de la Philosophie, en 1978, la creación del Collège International de Philosophie en 1983, y la fundación, al año siguiente, del Corpus des Oeuvres Philosophiques de Langue Francaise, son otros tantos indices de la transformación del ejercicio del pensamiento y de la invención de una nueva relación de los filósofos con los poderes. ${ }^{(9)}$

La coyuntura está marcada por la necesidad de filosofar de otra manera. Para comprender esta situación presente, Miguel Abensour proponía tomar en consideración cuatro elementos: la incapacidad reconocida de las ciencias humanas y sociales para dar respuestas satisfactorias a las inquietudes del presente; un despertar filosófico que no reivindica más la hegemonía en el campo de los saberes, sino que trabaja en la cuestión del sentido en un mundo de donde éste parece haberse ausentado definitivamente; la marcha del pensamiento contemporáneo, dirigiéndose hacia los límites del dominio filosófico y explorando dominios u objetos hasta entonces excluidos por la tradición; la emergencia, cada vez más fuerte, de la idea de un derecho a la filosofia para todos, correlativo de la libre expresión de los conflictos de pensamiento en el Estado social democrático.

Es por esto por lo que parecía que la filosofia es hoy portadora de un modelo político, el de una democracia igualitaria. En el fondo de toda tentativa filosófica se encuentra la idea de que el maestro es, en primer lugar, aquel que dice que no sabe, y que ignora todo, salvo que conviene buscar saber, cada uno por sí mismo, y todos juntos. ${ }^{(10)}$ Es así como nuestra generación ha pasado de la filosofia fuera de sí a la filosofia en común.

\section{Una historia política de la Filosofía}

Esta utopía reguladora de la filosofia viviente de hoy es lo opuesto a los ideales que presiden la institucionalización de esta disciplina en Francia a comienzos del siglo XIX. Pero ella asume, tal vez en la indignidad, esta herencia: en primer lugar, porque Francia es uno de los raros países en el mundo en los cuales la filosofia es, antes que nada, la materia de una enseñanza - obligatoria en la preparación del bachillerato que sanciona los estudios secundarios-y los filósofos son profesores funcionarios, es decir, profesionales asalariados que cumplen un servicio público bajo la autoridad del Estado. En seguida, lo que alli se enuncia bajo el nombre de filosofia - y esto es percibido con asombro, desde hace dos siglos, por aquellos que, 
al otro lado de la Mancha y del Atlántico, no tienen esta tradición y espontáneamente la mirarían como un uso ilegítimo de la filosofia - reivindica, si no ser prescriptivo para la política, al menos tomar la palabra en el momento en que interviene la cuestión de lo político. Finalmente - pero más adelante volveré sobre ello-, por el hecho de que la filosofia tiene el estatuto de una disciplina literaria y porque, a ese título, se autoriza para resistir a la ciencia. ${ }^{(11)}$

He partido de tres preguntas: ¿Cómo se constituye esta profesionalización de la filosofía que comienza con la Revolución y el Imperio se acaba, en su momento originario, en 1830? ¿Cuáles son las condiciones de posibilidad de un discurso de enseñanza filosófica? ¿Cuáles son las estrategias puestas en obra por la filosofia para llegar a la dominación ideológica en la Universidad y en el Estado constitucional moderno?

Fundada explícitamente sobre una triple exclusión: la de los sistemas filosóficos que la han precedido, la de las formas de sus enunciados y la de sus destinatarios, en razón de la función nueva - oficial y escolar- que le es atribuida, la doctrina que realiza esta institucionalización de la filosofia puede ser descrita, en 1847, en estos términos por Armand Fresneau: "Eclecticismo, filosofia de la Universidad, nueva filosofia francesa, todos términos sinónimos en el Diccionario de M. Cousin, cuya escuela se proclama la única escuela filosófica actualmente existente en Francia, y declara con razón, a quien quiera escucharle, que la Universidad le pertenece."(12)

Ella es el resultado de un proceso que precede al (re)nacimiento del espiritualismo en Francia y se origina, paradojalmente, en el proyecto de los Ideólogos en volver inmutables, mediante la enseñanza, los principios del orden social revolucionario, creando instituciones de educación, de formación y de investigación sometidas a la filosofia de Condillac, heredera de la de Bacon y de Locke: "el análisis es esencialmente un instrumento indispensable en una gran democracia; la luz que extiende tiene tanta facilidad para penetrar en todas partes que, como todos los fluidos, tiende sin cesar a ponerse a nivel".(13) Esta nueva relación de la filosofia con la enseñanza y con la política se desplaza bajo el Imperio, que modifica el cálculo, y la Restauración, que trastorna el paisaje especulativo. Con la ley de 1802 y el decreto de 1808, que introducen la lógica y la moral en el programa de los estudios secundarios, y el reglamento de 1809, que instituye un año de filosofia durante el cual los alumnos deben aprender, en francés o latín, los principios de la lógica, de la metafisica, de la moral y la historia de la opiniones de los filósofos, es establecido el dispositivo jurídico para una enseñanza que, en primer lugar, restaura el contenido de los viejos manuales de los colegios de Ancien Régime. La filosofía no es más esta ciencia nueva que, por su método aplicado a todos los órdenes de educación, forma para la libertad al pueblo de los ciudadanos de la República, se convierte en un saber de los primeros principios que garantizan la fidelidad al Imperio y en una materia para ser enseñada a los alumnos de los liceos solamente para la preparación del bachillerato. La Restauración reconduce, en 1814, esta 
legislación, y la completa por la instauración de una pregunta de filosofia en el concurso general (1817) y la creación de un premio de honor específico (1820), imponiendo el latín como lengua de los cursos de filosofia (1821), redefiniendo el programa del bachillerato (1823), creando una agregación de filosofia (1825). No obstante, estas disposiciones intervienen poco en la realidad, pues a menudo se obtiene el bachillerato sin que haya sido seguido el curso de filosofia. Y no es sino hasta después de 1830 que su programa se transformará, de manera decisiva, en un cuerpo de enunciados surgidos directamente del eclecticismo cousiniano, repartidos según cuatro divisiones: psicologia, lógica, teodicea, historia de la filosofia.

Al mismo tiempo que el Estado constituía a la filosofia como un saber, los filósofos eran conducidos de la filosofia a la política. Con Royer-Collard, y luego con Víctor Cousin, se ha elaborado, en primer lugar en el círculo restringido de la Escuela Normal -el de esa juventud estudiosa que formará a la generación siguiente en los conocimientos que son la base de las profesiones liberales e incluso en aquellos que son su ornamento-, (14) y luego en la Facultad de Letras de París, ante un público numeroso y muy pronto internacional, una filosofia percibida como nueva y que combate el sensualismo condillaciano, en primer lugar a nombre de sus efectos. En el discurso inaugural de su curso de 1813, Royer-Collard discute que los errores de la filosofia sean puramente especulativos: el escepticismo es destructor de las leyes de las sociedades, y de los derechos y deberes que constituyen la moral pública y privada. Víctor Cousin retoma la critica de esta deplorable filosofía, raiz de las desgracias de la patria, para definir finalmente su propia doctrina como fundando la verdadera libertad y legitimando la Carta constitucional.

De ahí la primera hipótesis de investigación, que me ha parecido la más evidente y la más fácil de poner a prueba: habría razones internas a una filosofia para desembocar en una política determinada. Cuestión de historia filosófica de la filosofia, que la filosofia contendría tan pronto como acepta una escansión histórica de su propósito, sea esta escansión un plazo temporal en el cual el lector (o el auditor) presumidos son pensados como accediendo a los sistemas de filosofia, o un corte de épocas a las cuales la filosofia se habría anudado, o una modalidad de la representación en la cual se dejaría figurar en razón una sucesión de las filosofias. A partir de esta escansión, una política de la filosofia no deja de ser reformulada por el propósito mismo de la filosofia. Así Henri Gouhier, por ejemplo, ha podido demostrar que "todo uso de la noción de progreso en filosofia opera una especie de inversión por la cual la historia de la filosofía se convierte en una cierta filosofia de la historia". (15) Y bajo este aspecto, no es de ninguna manera sorprendente sino, al contrario, perfectamente lógico comprobar que las filosofias tocan a lo político de la manera más esencial, precisamente en los momentos más esenciales de su propósito filosófico. Robert Flint y Martial Guéroult han consagrado a la historia de la filosofia y a la filosofia de la historia de Víctor Cousin(16) páginas prodigiosamente esclarecedoras. Pero esta percepción de lo político en las escansiones mismas del propósito filosófico plantea la cuestión de la escansión en la cual 
ella se deja percibir. Es evidente que, a este respecto, sería insuficiente ceder a la imagen, que se presenta espontáneamente, de una historia, génesis o políticas internas de la filosofia. Lo interno y lo externo, así como el conjunto de las categorías (racional/empírico, universal/particular, necesario/contingente, teórico/práctico, Histórico/histórico, etc.) susceptibles de reconducir su oposición, no parecen poder hacer justicia, en el seno de las políticas adelantadas por la filosofia, precisamente a esa política que se revela en el mismo propósito de la filosofia. Esas categorías corren el riesgo de subestimar la amplitud y la necesidad de la política en el seno de lo que ellas constituyen como una exterioridad de lo filosófico.

La segunda hipótesis, exactamente inversa a la precedente, sería la de partir de la política para encontrar allí la causa de la invención de un dispositivo especulativo adecuado para sus finalidades estratégicas. Esa es, por ejemplo, la lectura que ha intentado Lucien Sève en La Philosophie Contemporaine en France. (17) Confrontada con un vacio ideológico y en la imposibilidad de recurrir al campo de la reacción nobiliaria, monárquica y clerical - afiliada a Roma, a los Jesuitas u a los Ultra-realistas - para oponerse al peligro social de la filosofia de las Luces, portadora de la revolución, la burguesía habría creado de alguna forma, en el laboratorio político, una filosofia a la medida para la formación de las élites. Habría así dirigido, sucesivamente, la operación Escocia (un Sedán filosófico que sustituye la doctrina de Reid y la de Dugald-Stewart por nuestro gran siglo XVIII), con Royer-Collard; luego, con Víctor Cousin, la operación Kant (el filósofo del compromiso de la ciencia y de la religión); $y$, después de un rodeo por Hegel y el segundo Schelling, una operación pasado, que se supone reinscribe la nueva filosofia en la raíces francesas cartesianas. Esta historia externa de la relación de la filosofia con la política condujo a resultados decepcionantes, por lo que ella terminó por demostrar, para Víctor Cousin y la filosofia universitaria, lo que demuestra y demostrará de todo objeto: que es todavía una de las múltiples formas bajo las cuales la burguesía dirige una lucha idéntica a ella misma bajo todas sus figuras. Ella reduce a los actores a no ser más que políticos (y ciertamente es importante que ellos sean también hombres de Estado), pero no considera la especificidad de su condición filosófica.

Otra dirección de búsqueda, tercera hipótesis: ¿no se podría explicar este compromiso político de la filosofia por una necesidad de orden social? Esta opinión ha sido sostenida por Emile Durkheim. Para él, Víctor Cousin hubiera querido fundar una nueva iglesia que, "no reclamando de sus fieles sino sinceridad y algo de lógica, se encontró naturalmente con la parte sana de la nación y no tuvo que excomulgar sino a los espíritus falsos y a los corazones pervertidos". (18) Dos causas presiden este proyecto:

"Partiendo del hecho, de alguna forma legal, de que Francia comprendía cultos diferentes, y tal vez de este otro, menos oficial, de que la diversidad de las doctrinas particulares superaba todavía, y con mucho, la de los cultos reconocidos, Cousin concluyó que no era posible contar con ninguna religión establecida para asegurar la perpetuidad del espíritu nacional. De otra parte, él estaba penetrado de la idea de que 
ninguna sociedad es posible si sus miembros no tienen en común ciertas creencias fundamentales. El tuvo, entonces, el sueño de mantener esta indispensable comunidad de principios sin servirse de ninguna autoridad religiosa y no apoyándose sino en la mera razón".

Durkheim pudo jactarse con razón de las declaraciones del propio Cousin para apoyar su análisis: está comprobado que, en numerosos textos, éste reclama que la enseñanza filosófica se ponga al servicio de la unidad nacional, a partir de la homogeneidad postulada de las verdades reveladas por la religión y demostradas por la filosofia. Por lo demás, ¿se puede reducir la política de la filosofia de Cousin a su proyecto explícito? Si Durkheim encuentra aquí una nueva ocasión para probar lo bien fundado de su teoría de las funciones sociales y de las instituciones, y propone una interpretación general del eclecticismo que conviene tomar en consideración, no dice nada de los procedimientos concretos de producción del corpus de los conceptos que se autodesigna bajo el nombre de filosofia, sobre la formas materiales de existencia de esos discursos en la institución, sobre la tradición de la cual dependen, y sobre la distorsión entre lo real y lo ideal en la institución filosófica.

¿La manera de abordar la cuestión de la institución filosófica y de la relación de la filosofia con la política por los sociólogos contemporáneos, no procede por una reducción análoga, cuando trata a la filosofia como un producto simbólico entre otros, al campo filosófico como dependiendo de una doble pertenencia al campo universitario y al campo intelectual, y el compromiso político de sus agentes como el de cualquier otro intelectual? Además de las especificidad de lo filosófico, la teoría de la reproducción - esta crítica radical de una situación radicalmente inmutable aplicada a la filosofia- ${ }^{(19)}$ aparece aquí, como en otras partes, impotente para dar cuenta de las singularidades, para pensar las formas de resistencia y las prácticas subversivas de la institución de una manera distinta a ellas mismas, sometidas a las leyes de la autoridad legítima que denuncian y, por ello mismo, desconocen, y a aprehender el sentido de sus mutaciones y de sus devenires en su relación con lo político.

Ahora bien, como hemos visto, yo había llegado a Víctor Cousin y a su política de la institución por el rodeo de la contestación del orden filosófico establecido. Esta orientación fue reforzada por la lectura del panfleto de un profesor de filosofia suspendido en 1842 por haber dictado un curso sobre las ideas políticas de Platón y de Aristóteles en la facultad de Estrasburgo: Les Philosophes Salaries de Joseph Ferrari, publicado en 1849, y cuya reedición emprendí. Reconstituyendo su caso a partir de archivos, me había detenido en este juicio emitido en otra de sus obras, contemporánea de la precedente: Machiavel, juge des révolutions de notre temps.

"(Bajo Louis-Philippe) el culto del hecho cumplido tuvo adoradores, sicofantes, una policía; la filosofia tuvo sus esbirros cuyo jefe, adorador del éxito por método, impuso 
a la enseñanza una mezcla calculada de erudición y de bajeza, constituyéndose en el turiferario de la fábula y en el enemigo personal de todo libre pensador., ${ }^{(20)}$

Yo había intentado tomar a Víctor Cousin, a sus discípulos y al eclecticismo por el otro extremo de la cronología, cuando el general - según su propia expresiónreina sobre su regimiento y su filosofia sobre la Universidad, y en un momento fuerte de la impugnación de su legitimidad, o sea en una crisis de la institución filosófica. Y si la enseñanza filosófica podía ser pensada como el modelo de la formación de las élites bajo la monarquía constitucional, era necesario apelar a la historia para restituirla en su contexto político.

Como se ha visto, la enseñanza filosófica preexistia a Víctor Cousin, pero es verdaderamente con él que se codifica bajo esta forma bien francesa del coronamiento de los estudios secundarios, cuando él llega a ser, después de 1830, miembro del Consejo Superior de la Instrucción Pública, presidente de la agregación de filosofia, director de la Ecole Normale y presidente de la Academia de las Ciencias Morales y Políticas. La revolución de julio lleva al poder a una nueva clase política, los liberales, cuya figura ejemplar es Guizot. La primera preocupación de éstos, y singularmente del grupo de los Doctrinarios que reúne bajo la Restauración, además de Guizot, a Royer-Collard, Duvergier du Hauranne, y sus discípulos del Globo (Dubois, de Rémusat) es la de fabricar los cuadros del Estado, la "aristocracia legítima" de la sociedad liberal moderna. Con este fin, establecen un curso de formación a cargo de una casta de clérigos, los profesores de filosofia, destinados a la enseñanza de Estado, y que, por consiguiente, deben liberarse tanto de la tutela de la Iglesia como de toda influencia directamente política.

Para describir este fenómeno, recurrí a los historiadores americanos y al uso que ellos hacen del concepto de letrado, que pertenece a la sociología weberiana de la burocracia. En particular, el análisis de Fritz O. Ringer de la decadencia de los mandarines alemanes entre 1890 y $1923^{(21)}$ me parecía que podía aplicarse, por analogía, a la situación de Francia. Para Ringer, entre el Antiguo Régimen (donde los privilegios de nacimiento bastan y son necesarios para dirigir el Estado) y la sociedad industrial avanzada (que da prioridad a la competencia técnica de los individuos para la gestión de los asuntos del Estado y la concede a los detentadores del capital), hay un intervalo histórico que da el poder a letrados cuyo solo capital es la instrucción. La hipótesis me interesaba, en primer lugar, porque permitía singularizar grupos restringidos, sin disolverlos en las clases sociales. Podía ser descrita la constitución, en la primera mitad del siglo XIX, de un tipo ideal de intelectual cuya figura ejemplar es la del profesor de filosofia; Ringer va a buscar su modelo en el mandarín chino tal como lo describe Max Weber, o sea una élite social y cultural que debe su estatuto a cualificaciones que derivan de la formación y de la cultura más que de derechos hereditarios. Én este estado intermedio entre la propiedad de bienes raíces y la fase económicamente desarrollada, el capital cultural y el estatuto profesional está momentáneamente en posición de rivalizar 
con el prestigio cultural de la aristocracia de nacimiento y la cualificación social. Los mandarines letrados constituyen una verdadera nobleza sabia, una aristocracia de funcionarios, que participa en todos los procesos decisionales del Estado y ocupa un empleo en la administración pública. Yen esta fase donde el monarca constitucional intenta extender su poder, él confia a las élites cultivadas el cuidado de hacer su política, y es a nombre de sus saberes que aquellas pueden asi autorizarse, en lugar de la nobleza de nacimiento, para gobernar: "El Estado no existe ni por los dirigentes ni por los dirigidos, sino solamente a través de los hombres de cultura y de su saber".

Así he llegado a considerar, cuarta hipótesis, que la institución filosófica debia ser constituida como un objeto privilegiado de la historia del Estado moderno, y esto no solamente porque los principales filósofos franceses de la primera mitad del siglo XIX tienen una doble pertenencia a los cuerpos universitario y político - Cousin y Jouffroy son profesores y miembros de las cámaras-, sino también, y en primer lugar, en tanto que la edificación de la ciencia, y singularmente el discurso filosófico, forma parte de la constitución del Estado.

$\mathrm{El}$ acceso a esta cuestión por la lectura de Ferrari y la reconstitución de su caso me confirmaba en esta vía, a partir del momento en que me di cuenta que él no era el único en ser víctima de su práctica de la enseñanza filosófica, sino que muchos otros profesores de filosofia - tales como Charma en Caen, Bersot en Bordeaux, Bouillier en Lyon, Zévort en Rennes-, sufrian, ellos también, entre 1842 y 1844, los ataques reiterados del "partido-sacerdote". Aquel, tomando como blanco a la filosofia universitaria por impugnar el monopolio de la enseñanza, ponía en causa la legitimidad del poder de las letras bajo la monarquía de julio. La constitución de esta élite podía, por lo tanto, ser ubicada a través de estas crisis de la institución filosófica. Según Kirchheimer, (22) la crisis es el momento determinante en el que todos los retos ocultos de una institución aparecen: o sea el momento político por excelencia, donde se funda la distinción entre amigos y enemigos - distinción problemática si se la refiere a Carl Schmitt, por lo que prefiero entenderla con Guizot-. Intentaba, entonces, trabajar las crisis, con el fin de ilustrar un proceso decisional en un momento donde se revelan en caliente los desafios de la institucionalización de la filosofia en el seno del Estado constitucional.

A partir de este estudio era posible establecer una tipografia sumaria que distingue: crisis de la institución filosófica donde la oposición comparte la misma ortodoxia que el poder mandarinal: sobre la base de esta ortodoxia compartida, la oposición nace de una diferencia de posición en la pugnacidad y la oportunidad del combate con relación a la Iglesia; y crisis que conciernen centralmente al antagonismo de la institución filosófica y de la Iglesia. En las primeras, algunas apuntan como reto al reconocimiento del candidato por la institución, y el resultado de ello era ya la sumisión, ya la impugnación de los profesores (Charma, Bersot); otras tomaban frontalmente por objeto la ortodoxia de la doctrina, o sea el momento en que ésta se constituye como tal (Bouillier). Las segundas se desarrollan en dos 
etapas: en primer lugar, hay enfrentamiento con un polo exterior, luego lucha en el interior de la institución (Zévort, Ferrari). Pero se es llevado de nuevo al caso de la figura precedente, porque se trata siempre de la constitución y de la defensa de la ortodoxia. Es este trabajo el que constituye la segunda parte de este estudio.

Esta descripción de la institución filosófica separa un cierto ideal de la libertad de filosofar de una enseñanza conforme a lo que se constituye como una política de la filosofia a través de esta serie de crisis que separan los buenos de los malos discursos, que no conviene pronunciar en una cátedra universitaria. A partir de allí se determinan normas explícitas e implícitas de la función de profesor asalariado. Con ellas aparece, tal como evoca Joseph Ferrari, la figura del filósofo sin conciencia (que se ha vendido al poder político de Louis-Philippe por el dinero, los honores y una vida fácil). Pero, por encima de esta descripción, se planteaba la cuestión de las condiciones de posibilidad de la constitución de espiritualismo universitario como doctrina oficial de la monarquía constitucional.

Es convenido, a partir de la historiografia del siglo XIX, distinguir dos períodos en la vida y en la obra de Víctor Cousin: antes de 1830, intentará producir una filosofia nueva; después, abandonará toda pretensión teórica para consagrarse a la gestión de su política de la filosofia, contentándose únicamente con reescribir sus cursos y sus artículos antecedentes para adaptarlos a dicha gestión. El punto de vista que adopto, o sea el de una historia inextricablemente política y filosófica de la filosofia, me hará sostener una tesis casi opuesta. Víctor Cousin hereda de Royer-Collard un proyecto que no es solamente teórico, sino también, desde el origen, institucional, que modifica hasta darle el estatuto de una tradición filosófica nacional y la función de un paradigma político del Estado liberal constitucional. Ese proceso obedece, por consiguiente, a dos lógicas estrechamente imbricadas, una lógica filosófica y una lógica política. El eclecticismo no es percibido más como una doctrina primero constituida, que luego sería sometida a fines no filosóficos; él participa, tanto en su modo de elaboración como en su arquitectura conceptual, del paradigma político. En este segundo sentido, es un objeto de derecho irreductible a una historia puramente doctrinal, como las elucidaciones marxistas o sociológicas que tomaban simplemente por objeto de estudio el reverso, el lado oculto, lo otro inmediato de la doctrina. El surge, más bien, de una aprehensión de la filosofia que recuerda a ésta la cuestión de su relación con el Estado, sosteniendo, al mismo tiempo, la imposibilidad de pensar plenamente al Estado, tanto como a la filosofia, fuera de la filosofia.

El eclecticismo, que contiene en lo más profundo de sí mismo la cuestión de la imposibilidad de pensar por fuera de la filosofia la formación del Estado moderno en Francia, debe ser pensado en todo el rigor de esta cuestión. Ella autoriza, en consecuencia, a constituir la filosofia de Cousin como sistemática ( $\mathrm{y}$, por consiguiente, a pensar sus discordancias y sus inconsecuencias internas como formando parte del orden de sus razones), y a prestar tanta atención a la propia obra de Cousin como a los comentarios de sus discípulos y de sus críticos, en lo que los une 
más bien que en lo que los separa. O sea, a describir, a partir de los discursos que polemizan en ese campo agonístico restituido, la configuración general de una política de la filosofia. Una manera de demostrar que la matriz ecléctica está sometida a un modelo propiamente político es cuestionar la reivindicación cousiniana de producir una filosofia para el tiempo presente.

\title{
Una Filosofía imparcial para el tiempo presente
}

El 22 de mayo de 1830, en un artículo que titula Des mots à la mode, Balzac ataca a Víctor Cousin y a sus discípulos eclécticos:

\begin{abstract}
"¿Se habla de filosofia? Oh! Quien quiera que usted sea, considere que si no sigue atentamente la moda, puede estar perdido para siempre, sirviéndose de palabras que terminan en té, como objectivité, subjectivité, identité, simultaneité, fugitivité, cuando el maestro ha hablado por isme, empleando las palabras sensualismo, idealismo, dogmatismo, criticismo, budismo, etc... O si usted toma los ismos cuando él pone en la boca los ion, como afección, sensación, inspiración, argumentación, usted puede pasar por un tonto. Por ejemplo, durante los equinoccios le es permitido envolver su pensamiento en frases como esta: La potencia de reproducción de la reflexión no se extiende hasta ciertos fenómenos, porque si la reflexión es una totalidad, es una totalidad confusa. Pero los días de luna llena, usted debe tomar un lenguaje más claro y decir: El sensualismo se hunde por la sensación en el mundo sensible."(23)
\end{abstract}

Cuestiones de palabra y de moda a la víspera de los Tres Gloriosos, la juventud letrada, enamorada de libertad, se reconoce en palabras huecas que sirven de estandarte a los intereses o a las pasiones más alejadas de la verdad. Los pensadores a la moda de la nueva filosofia de París - la expresión es de Stendhal- no hacen otra cosa que rebatir con fuerza palabras de los sistemas antiguos: siembran granos sin germen, expresiones que, según Balzac, no responden a ninguna idea. ${ }^{(24)} \mathrm{Se}$ mantienen a distancia de los hechos.

Palabras más bien que hechos, sin consideración de la teoría que quiere aplicar el método experimental a los hechos de conciencia y hace entrar, por consiguiente, las ideas, operaciones y modificaciones de las almas, y hasta las percepciones puras, en el rango de lo que es observable. Palabras oscuras que ocultan el vacío de un pensamiento detrás del cual se disimulan un simple retorno al espiritualismo y un proyecto político reaccionario, sin dirigir la atención a la invención, de la cual es portadora la importación de las doctrinas escocesa y alemana en Francia, y a la originalidad de la constitución de una relación específica de la filosofia y del Estado en la sociedad post-revolucionaria. Esta crítica arraiga directamente sobre aquella de los partidarios de la ideología y de la fisiología quienes, como Stendhal y Broussais, ${ }^{(25)}$ no veían en la Escuela de Cousin sino una 
tentativa retrógrada que conduce a la élite más acá de la ciencia y de la Revolución Francesa, y niega a su doctrina la dignidad de una filosofia presente.

En oposición a esta imagen, Víctor Cousin intenta acreditar la de una investigación que se hace a cargo de las cuestiones filosóficas del tiempo, y produce una filosofia original: un eclecticismo imparcial aplicado a los hechos de conciencia, que esclarece toda la historia de la filosofia y es demostrada por esta misma historia. (26) Imparcial, por supuesto, por el rechazo a condenar completamente cualquier sistema que sea, porque ninguno es falso, sino que todos, pese a su pretensión a la exclusividad, son incompletos y no agotan los fenómenos de la conciencia. Imparcial se dice también de una posición que se quiere más allá o por encima de los partidos singulares, de una legitimidad para decidir sobre la división de lo verdadero y de lo falso a nombre de la verdad una y universal.

La metáfora política corta el hilo de todas las descripciones que dibuja Cousin de la escena filosófica:

"Proponed entonces a los partidos, os lo ruego, deponer sus pretensiones tiránicas en el servicio de la patria común. Todos los partidos os acusarán de ser un mal ciudadano. Las doctrinas exclusivas son en la filosofia lo que los partidos son en el Estado. El eclecticismo tiende a sustituir su acción violenta e irregular por una dirección firme y moderada, que emplee todas las fuerzas, no desdeñe ninguna, pero que no sacrifique a ninguna el orden y el interés general". (27)

En el prefacio de su traducción del Manual de Filosofia de Tennemann, fechado el 1 de setiembre de 1829, Cousin propone una interpretación de la coyuntura filosófica cuyo modelo es directamente importado de aquelia que se hacen de la situación política del tiempo los liberales constitucionales. Describe tres vías que puede escoger la filosofia del tiempo presente. La primera es imposible de adoptar. Es la de la abdicación: la renuncia a la independencia, la sumisión a la antigua autoridad, el retorno a la Edad Media. Esta imposibilidad reside en la naturaleza misma de la filosofia, definida como representación de la civilización moderna. Por eso ella no podría ir a contrapelo del progreso general de la independencia y de la secularización de todas las cosas, Estado, ciencia, arte, industria. De esta definición cuasi hegeliana de la filosofia, Cousin extrae el argumento para invalidar a la escuela teocrática: si la naturaleza de las cosas progresa en un sentido determinado, allí está la voluntad de la Providencia, y la civilización y la filosofia no podrían ir en sentido inverso. Vuelve la argumentación de los teócratas contra ellos mismos: es con la razón que ellos atacan a la razón, y su llamado a la fe ciega no es sino un paralogismo pusilánime.

La segunda vía - dejar a la filosofia en el estado en que los siglos XVII y XVIII la habían dejado- es también imposible. Pues no es posible detenerse en el camino, y renunciar a la completud, mientras que en todas partes, en política como en las artes y en la literatura, se aspira a ella. Ninguno de los cuatro grandes sistemas que la filosofia moderna reproduce y que, desde el comienzo, Cousin ha mostrado 
ya en obra en sus cursos de la Sorbona: el idealismo, el sensualismo, el escepticismo y el misticismo, es enteramente verdadero, $y$ todos contienen intolerables extravagancias que son conocidas. Todavía aquí el modelo es político: de la misma manera que todos los regímenes políticos, de la monarquía absoluta a la democracia igualitaria, han probado a qué funestas consecuencias podían llevar, así mismo las figuras de la filosofia han manifestado sus aterradores efectos, y su incapacidad para alcanzar la verdad absoluta.

La tercera vía: no rechazar ningún sistema y no aceptar ninguno entero, queda como la única razonable. O sea el eclecticismo, tratado de paz sobre la base de concesiones recíprocas entre los partidarios fanáticos de los sistemas exclusivos.

Allí donde Balzac y muchos otros no ven sino un fenómenos superficial - la búsqueda, por algunos jóvenes filósofos liberales de cabeza débil y corazón caliente, de una posición social dominante por la publicidad-(28), Víctor Cousin da a la constitución del campo agonístico en el cual se sitúa un estatuto propiamente filosófico. Si ha querido entrar en la pelea fue, escribe, para forzar al último sistema exclusivo, el sensualismo, a descender de su trono y a aceptar el derecho de examen. La lucha se ha extraviado, a falta de un adversario a su altura, en las declamaciones y las calumnias, cuando él esperaba objeciones.

Se propone, en consecuencia, asegurar a la vez la defensa y el ataque, pues "no hemos entrado en la ruta en que nos encontramos para recibir aplausos, sino para servir allí a la filosofia". O sea un camino señalado que la reflexión y el estudio no pueden tomar sino escogiendo la equidad, la moderación, la imparcialidad, la sabiduría, es decir, la tolerancia filosófica. No se trata de destruir a la filosofia del siglo XVIII sino de ponerla en su lugar, como es necesario hacerlo con cualquier otro sistema en la historia de la filosofia. De esta última dice Cousin que es una lección perpetua de eclecticismo:

"no hay nada que hacer sino honrar al espíritu humano, respetar su libertad, comprobar las leyes que la regulan y los sistemas fundamentales que emanan de esas leyes, perfeccionar sin cesar esos diversos sistemas uno con el otro, sin intentar destruir ninguno, buscando y desprendiendo la porción de verdad que cada uno de ellos encierra, y por la cual cada uno de ellos es hermano de todos los otros e hijo legítimo del espíritu humano".

Lejos de ser un retorno al pasado, el eclecticismo se quiere una filosofia del presente, que apunta a la emancipación de la tradición y postula, a la manera hegeliana, una identificación del pensamiento filosófico y del pensamiento de la época.

La primera lección del Curso de 1828 estaba enteramente consagrada a probar esta tesis, según la cual la filosofia, necesidad real y producto necesario del espíritu humano, es el último desarrollo y la última forma del pensamiento, la luz de todas las luces y la autoridad de todas las autoridades, lo propio de la época. 
"La filosofia es la inteligencia absoluta, la explicación absoluta de todas las cosas. ¿De qué, entonces, podría ser enemiga? La filosofia no combate a la industria, sino que la comprende, y la vincula a principios que dominan a aquellos que la industria y la economía política reconocen. La filosofia no combate a la jurisprudencia, sino que la eleva a una esfera superior; ella hace el espíritu de las leyes. La filosofia no corta al arte sus alas divinas, sino que lo sigue en su vuelo, mide su alcance y su objetivo. Hermana de la religión, toma, en un comercio intimo con ella, sus aspiraciones poderosas; aprovecha sus santas imágenes y sus grandes enseñanzas pero, al mismo tiempo, convierte las verdades que le son ofrecidas por la religión en su propia sustancia y en su propia forma; no destruye la fe: la esclarece y la fecunda, y la eleva suavemente del amanecer del símbolo a la luz del pensamiento puro". (29)

Hegel no dice otra cosa afirmando que la filosofia como conocimiento del ser es, al mismo tiempo, "su época aprehendida en el pensamiento".(30)

$\mathrm{Y}$ en este sentido, la interpretación que hace Joachim Ritter de la teoría hegeliana se aplica exactamente a Cousin: si la modernidad está radicalmente por fuera de la tradición filosófica, si la cuestión es más bien aquella de la esencia del presente histórico-político y de su verdad en tanto que él hace ruptura con el pasado, $y$, por consiguiente, es la de la metafisica en tanto que ella rompe con su propia historia, entonces el problema se convierte en el de la emancipación. Como para Hegel, el acontecimiento de la Revolución Francesa es el punto nodal de constitución del campo de la cuestión.

El intérprete moderado, pero inquebrantable, de la Revolución Francesa

El $1^{\circ}$ de abril de 1851 , Víctor Cousin da a la Revue des Deux-Mondes un artículo sobre los principios de la Revolución Francesa y del gobierno representativo, que servirá de prefacio a la edición de sus Discursos Políticos. Artículo coyuntural, porque no se trata de tomar posición con relación a los acontecimientos de febrero de 1848 y a la contrarrevolución que se abre paso. Pero más allá de esto, este texto tardío puede ser tenido por un manifiesto en el cual Cousin hace la confesión de su apego y de su fidelidad a una cierta idea de la Revolución Francesa, y puede esclarecer su política de la filosofia. Ciertamente, él puede con razón ser sospechoso de dar una lectura suplementaria, una vez más corregida y mejorada, de su recorrido: ¿acaso, en cada nueva edición de sus lecciones y de sus Fragmentos, no ha reescrito y atenuado, en función de las circunstancias, sus enunciados más audaces? Pero, al término de sus piruetas y de sus vuelcos, Cousin hace aquí, sin duda, la confesión del destino de la ruta sinuosa que él ha tomado. Su vida comienza con la Revolución Francesa, y no se ha consagrado sino a comprenderla y a amarla: mi filosofia no ha sido sino la reflexión aplicada a sus instintos y el resumen de sus máximas. ${ }^{(31)}$

En el orden de lo filosófico, la Revolución comienza con Descartes, quien por método parte del hombre para llegar al todo, incluso a Dios. Después de él, la razón 
se ha emancipado de la autoridad en provecho del libre estudio del pensamiento. La conciencia es la fuente de la moral, la que, por consiguiente, es una institución natural. Y como la política es hija de la moral, no puede seguir fundándose en alguna autoridad exterior. En el orden de lo político, los hombres, y sólo ellos, pueden aceptar el poder -o sea el principio de la soberanía nacional-, que responde a aquel de la soberanía de la razón en filosofia, y que la Revolución Francesa ha proclamado.

La época presente culmina esta doble revolución en la inteligencia y en el mundo. En ambos lado, la resistencia a lo nuevo se hace con los mismos paralogismos. Se acusa a la filosofia de llevar al escepticismo y al ateísmo, mientras que la razón emancipada no ha perjudicado a la causa de Dios, sino que la ha servido; el sentimiento y la fe no se oponen a la razón: el primero es una forma ingenua de ésta, la segunda viene a probar el resultado de un procedimiento racional. Los mismos pretenden que la soberanía nacional conduce a la anarquía; pero confian el gobierno, a nombre de Dios, a hombres contra los cuales se podrían sostener los mismos reproches con los que se abruma a aquellos cuya legitimidad proviene del pueblo: la monarquía absoluta no ha producido menos excesos que la Revolución Francesa. Y si esta demostración a priori de la absurdidad lógica de la posición contrarrevolucionaria no bastara, Cousin añade un argumento de hecho: por toda Europa, en esta primera mitad del siglo XIX, la Revolución Francesa ha sido imitada, señal cierta de la fuerza de su idea.

No obstante, Cousin no se detiene en la Revolución Francesa. El muestra que sus excesos fueron necesarios, porque todo progreso en la historia engendra, ineluctablemente, sufrimientos - como por ejemplo, la revolución cristiana-. La Revolución debía, en primer lugar, destruir el antiguo orden monárquico. Ella acaba un proceso. Después de ella, la tarea del siglo XIX será construir la sociedad moderna sobre la base de los principios que ha enunciado. Considerando a la sociedad como una persona moral, ha postulado la libertad y la soberanía de la nación. Y le ha dado los mismos deberes que al hombre: aquel, imperativo y absoluto, de la justicia - el mantenimiento y la defensa de nuestra libertad-, y aquel, facultativo en tanto que fundado en la virtud, de la caridad. El genio de la Revolución Francesa actúa, entonces, en estos tres principios universales: soberanía nacional, emancipación del individuo o justicia, fraternidad o caridad civil - lo que no significa en ningún caso derecho a la asistencia-.

La cuestión política de Cousin se convierte, en consecuencia, en la de la forma de gobierno que conviene hoy para la realización de esos principios, o sea la de la mejor organización del lazo social en una sociedad post-revolucionaria. Por haber sido escogida históricamente en Francia, en 1792, la forma republicana no se impone necesariamente como efecto de los principios de la Revolución Francesa. Ella es, más bien, un avatar coyuntural, la revolución en estado de crisis, el lado siniestro de la revolución, una negación. Puede convenir a una sociedad naciente, como América. Pero no podría ser necesaria para una gran nación civilizada, en un 
país europeo como Francia, que debe vincular su porvenir a su pasado, y debe conciliar el orden y la libertad:

"La monarquía constitucional es el gobierno verdadero de Francia y de Europa en el siglo XIX. Es el único que realiza la soberanía del pueblo con verdad y sin conmoción, con la ayuda de un rey que no mata y no hace daño, y con ministros responsables que cambian a merced de la mayoría de un parlamento que representa a la mayoría de los electores, la cual, a su turno. representa a la mayoría de la nación, de suerte que, en último análisis, es la nación la que gobierna, quiero decir la verdadera nación, inteligente e ilustrada, y no la masa ignorante, una veces indiferente y otras agitadas. Este bello gobierno admite, sin duda, una multitud de diferencias, según los países y las circunstancias, pero tiene un tipo más o menos uniforme que expresa la unidad de la sociedad europea".

Cousin lee la historia de Francia, en este medio siglo, como la continuidad de la exigencia de ese principio regulador. El Imperio, la Restauración, la Monarquía de julio, no han sido sino formas de gobierno conformes al espíritu moderado de la Revolución Francesa, y la caída de Napoleón, las de Carlos X y de Louis-Philippe, no han tenido por causas sino sus propias faltas, o sea haber derogado este espíritu que une los principios de orden y de libertad. La verdadera democracia, la mejor de las repúblicas, es, por lo tanto, la monarquía parlamentaria.

Este tardío escrito político de Víctor Cousin reafirma los principios de Justice et Charité, (32) publicado en 1848: el verdadero mundo del hombre es aquél de la libertad, su verdadera historia es la del progreso constante de la libertad, pasando del pensamiento a los derechos reconocidos y respetados: o sea la época última donde, por así decir, la esencia misma de la libertad se manifiesta. Pero va más lejos, porque demanda una forma política específica de gobierno para Francia y Europa, cuando en el otro escrito la elección había sido dada sin precisión, como asunto de lugar y de tiempo, sin duda para evitar toda polémica con los republicanos. Mientras tanto, se trata alli de la misma filosofia política que se escuchaba en las lecciones de 1828 y 1829 en la Facultad de Letras de París.

Si la cuestión implícita de la filosofia espiritualista francesa en la primera mitad del siglo XIX es: ¿cómo salir de la Revolución Francesa?, es decir, ¿cómo construir una sociedad post-revolucionaria?; habría que describir cómo, desde el punto de vista de la lógica interna del sistema cousiniano, se pasa de la idea de libertad a la de necesidad, y reencontrar el razonamiento que autoriza a una filosofia de la historia para legitimar una forma estatal singular como manifestación de la esencia de la libertad - sobre este último punto, Hegel pudo haber sido directamente influenciado por los franceses en lugar de ser su fuente. 


\section{La naturalización francesa de la metafísica alemana y el renacimiento nacional de la alta filosofia}

Las analogías de estructura entre el razonamiento de Cousin y el de Hegel son manifiestas, y numerosos contemporáneos lo habían percibido. Pero es necesario reconocer con Martial Guèroult que - si en los dos casos la historia de la filosofia es la más alta de todas las historias y la conciencia de la historia de la humanidad y de la naturaleza, y si hay identidad de la filosofia y de su historia, no siendo aquella sino el desarrollo necesario e inmanente de ésta- para Hegel ese proceso desemboca en una doctrina original que suprime las del pasado en su provecho, reconstituyéndola a priori, mientras que en Cousin la filosofia del tiempo presente es inducida a posteriori de la historia de la filosofia, concebida como una experiencia susceptible de ser observada, comprobada y combinada en sus elementos esenciales y verdaderos.

Siendo insostenible la pura posición ecléctica, por no proporcionar ningún criterio de separación entre lo verdadero y lo falso en las filosofias pasadas, Cousin se refiere finalmente a un espiritualismo que transige entre el método experimental a la manera de la filosofia del siglo XVIII francés y el método especulativo de la metafisica alemana. Y Guèroult tiene razón en afirmar que ese sistema no tiene nada que ver con el de Hegel y el de Schelling, y todavía menos si se considera el lazo entre la psicologia, el sistema y la historia de la filosofia: o sea, el hecho de comenzar por el análisis psicológico y hacer del análisis histórico la contraprueba de aquél.

Esta comprobación, en tanto que separa a Cousin de la tradición metafísica alemana, no hubiera disgustado a éste, que intentaba vanamente hacer olvidar sus recuerdos de Alemania, cuando estaba, según su propia confesión en 1861, "todavía demasiado cerca", en 1828 ,

"para que las grandes generalizaciones y las fórmulas un poco altaneras a las cuales estaba acostumbrado no destiñeran un poco, si se me acepta esta expresión, sobre mi pensamiento y sobre mi lenguaje, $y$ es posible que mis palabras algunas veces hayan presentado, a los espíritus prevenidos o poco familiarizados con estas materias, la apariencia de una doctrina bastante favorable al panteísmo. Pero, ciertamente, nunca esta apariencia estuvo más lejos de su realidad...... Yo tenía la costumbre, incluso con $\mathrm{M}$. Hegel y sus más devotos discípulos, de licenciarme un poco a cuenta de ese famoso Ser en sí, das reine Seyn, puro de toda determinación, y que por una serie de metamorfosis maravillosas se convierte en el principio de toda determinación, tanto de la calidad como de la magnitud". (33)

Pero, en tanto que comprobación, es tan poco evidente a los contemporáneos que Hegel mismo puede pretender que Cousin, así los haya ahogado en su salsa, le ha tomado algunos pescados. Los católicos, del abate Bautain al abate Maret, lo 
acusan de "hablar la lengua de Hegel"(34) y de haber tomado su "ruta funesta", incluso si "el eclecticismo no es el germanismo". Pierre Leroux escribe en 1841:

"M. Cousin, quien ha copiado y firmado (Hegel) en Francia, y que le había tomado todo, no obstante sin comprenderlo a fondo, tomaba también al Cristianismo bajo su protección. Aceptar todo, explicar todo, respetar todo, he aquí cuál era la divisa del Fatalismo en Berlín y del Eclecticismo en París.....Es así como la filosofía se había hecho real y aristocrática en Berlín y en París". (35)

YVéra cuenta que, aunque habiendo conocido muy poco su filosofia, Cousin en su juventud ha desbordado de entusiasmo por Hegel y su enseñanza, por lo que hoy (1865):

"Alemania ha llegado a ser (para él) una plaga, una úlcera que lo roe y no le da tregua, y que los nombres de Schleiermacher, de Schelling, de Hegel — sobre todo de Hegel-, se levantan ante él como la sombra de Banquo, y transforman en sueños espantosos, en pesadillas, sus más rientes visiones". ${ }^{(36)}$

Que Cousin haya sido fuertemente influenciado por la metafisica alemana, y singularmente por la filosofia de Hegel, es algo que, por la lectura de sus cursos a partir de 1818, y sobre todo por el de 1828, no se podría negar. Que haya intentado después, por razones estratégicas debidas a los ataques de los que la filosofia de la Universidad era objeto, fabricar la leyenda de una inspiración puramente francesa de su escuela y reducir la parte de la naturalización del pensamiento alemán en su enseñanza, ${ }^{(37)}$ se lo puede también demostrar. Pero cuando Cousin pretende, en 1845, que desde su primera enseñanza de 1815 a 1820 no ha hecho sino defender los principios de la psicología y de la teodicea cartesianas contra los escoceses y los alemanes, y que en su segunda enseñanza, de 1825 a 1830 , no se ha limitado a glorificar en todas partes el nombre y el genio de Descartes, sino que se ha consagrado a sacar a la luz el carácter general, la continuación, el progreso, los méritos, como también los defectos de la escuela entera, desde su fundador hasta Leibniz inclusive, haciendo de éste el último y más grande de los cartesianos, ${ }^{\left({ }^{(3)}\right.} \mathrm{o}$ sea la idea de que Descartes es el padre de la filosofia moderna de la cual Cousin es portador, no está lejos de fabricar sus propios textos.

Sobre este último punto, no se trata aquí de definir la naturaleza de "la estafa" de Cousin, en el sentido en que Jules Barbey dAurevilly entiende, en 1863, este término aplicado a la importación de la metafísica alemana en Francia: "En filosofia, es un pobre quien ha estafado los hábitos....... Cousin es el hazmerreír y el desprecio de Alemania. Ha ido a pedir limosna a la puerta de Hegel, y él se la ha dado, y ha regresado para hacer, con algunos centavos de Hegel, moneda falsa en París". (39) Se trata, en cambio, de cuestionar los desafios del (re)nacimiento de la tradición filosófica francesa en sus préstamos a otra tradiciones nacionales. Víctor Cousin había refutado desde 1833 la acusación lanzada contra él de haber importa- 
do la filosofia alemana y de haber introducido lo extranjero en el corazón de su país con el argumento según el cual en filosofia no hay otra patria que la verdad, $y$ reivindicando su propia no-originalidad: "Lejos de pretender que no haya tenido maestro, confieso que he tenido muchos, en el pasado y en el presente, y en Francia y fuera de Francia". (40)

Es, en consecuencia, al sistema material de estos préstamos y a las formas de su reapropiación, para la constitución de un dispositivo especulativo apuntando a la legitimación en el Estado constitucional moderno en Francia, que conviene también interrogar.

\section{Notas}

(1) Stéphane Douailler y Patrice Vermeren, “De l'hospice à la manufacture: le travail des enfants au XIX siècle", en: Les Révolles Logiques, N²3, Solin, 1976.

(2) Gustave Vapereau, "De la colonie agricole et penitentiaire de Meturay", en: La Liberté de Penser, $\mathrm{N}^{2} 4$, 15/05/1848, pág. 10.

(3) Stéphane Douailler y Patrice Vermeren, "Les prisons paternelles ou le grand air des enfants paurres", en: Les Révoltes Logiques, N 8/9, Solin, 1979.

(4) Ver, por ejemplo, Olivier Roy, "Les Etats Généraux ou l'apologie de nous-mêmes par nous-mèmes", en: Le Doctrinal de Sapience, $N^{2} 6$, Solin, 1979.

(5) En esta biblioteca del Doctrinal de Sapience, publicada en Solin, han aparecido: Défense de l'Université el de la Philosophie de Victor Cousin, presentado por Danielle Rancière (1977); y La Philosophie dans le mouroir, por el grupo de trabajo de profesores de filosofia en escuelas normales (1878), que hará aparecer después Les Crimes de la Philosphie. Recherches, N 49 (1979); "La présence de la philosophie dans les écoles normales et son historie", en: Cahiers Philosophiques, N² , abril, 1981; y La grève des philosophes, Osiris, Paris, 1984.

(6) Esta cuestión ha sido la materia de muchos seminarios en el Collège International de Philosophie, entre 1985 y 1988, adelantados solo o con la colaboración de Stéphane Douailler, Christiane Mauve, Georges Navet y Jean-Claude Pompougnac. Ver: La Philosophie saisie par l'Etat, Aubier, Paris, 1989.

(7) Joseph Ferrari, Les Philosophes sulıtiés, prefacio de Stéphane Douailler y Patrice Vermeren, Payor, Paris, 1979.

(8) Miguel Abensour, "Pierre Leroux et l'utopie socialiste", en: Eronomies et Soriettés, N 12, 1972, pág. 2201 yss.

(9) Francois Châtelet, Jacques Derrida, Jean-Pierre Faye, Dominique Lecourt, Rappont sur la fondation d'un college international de philosophie, Paris, 1983, retomado parcialmente en Jacques Derrida, Du droit a la Philosophie, Galilée, Paris, 1990.

(10) Jacques Rancière, “Comment savons-nous que nous sommes si critiques?”, en: La Grève des Philosophes, op. cit., pág. 119.

(11) Jean-Claude Milner, citado en Stéphane Douailler y Patrice Vermeren, "Corpus des oeuvres philosophiques de langue françaises", Universalich, 1989.

(12) Armand Fresnau, L'Eclectirisme, au Comptoir des imprimeurs-unis, Paris, 1847, pág. 9. 
(13) Ver Stéphane Douailler y Patrice Vermeren, "L'Institutionnalisation de l'enseignement philosophique français", en: L'Univers philosophique, bajo la dirección de André Jacob, t. I, P.U.F., Paris, 1989.

(14) Paul Gerbod, "L'Université et la Philosophie de 1789 a nos jours", en: Histoire de l'enseignement de 1610 a nos jours, Actes du 95 Congrés National des Societés Savantes, Reims, 1970, Bibliothèque Nationales, Paris, 1974, págs. 237 ss.

(15) Henri Gouhier, La Philosophie d'Auguste Comte, Vrin, Paris, 1987 (reedición); La Philosophie et son Histoire, Vrin, Paris, 1947.

(16) Robert Flint, La Philosphie de l'Histoire, Germer-Baillière, Paris, 1878, pág. 171 y ss.; Martial Guéroult, Histoire de l'Histoire de la Philosophie, Aubier, Paris, 1988, t. III, págs. 711-735.

(17) Lucien Sève, La Philosophie Française contemporaine et sa genèse de 1789 a nos jours, Editions Sociales, Paris, 1962, pág. 112 y ss.

(18) Emile Durkheim, "L'Enseignement philosophique et l'agrégation de philosophie", Rerue Philosophique, N 39,1895 , págs. 124-125.

(19) Jacques Rancière, "L'éthique de la sociologie", en: LEmpire du Sociologue, La Découverte, Paris, 1984, pág. 27.

(20) Joseph Ferrari, Machiusuel, juge des rèvolution de notre lemps, Joubert, Paris, 1849, pág. 117.

(21) Fritz O. Ringer, The Decline of the Germun Mandarins, Harvard University Press, 1965.

(22) Kirchheimer, Politics, Laws, Justice, New York, 1969, págs. 37-74.

(23) Honoré de Balzac, "Des mots a la mode", en: La Morle, 22/05/1830; se trata aquí del curso dictado por Cousin en 1829.

(24) Honoré de Balzac, "Les complaintes satiriques dans les moeurs du temps présent", en: La Mode, 20/02/1829. Ver Max Andréoli, "Balzac, Cousin et l'éclectisme", en: L'année balzacienne, Garnier, Paris, 1971.

(25) Broussais, De l'irrilation et de la folie, J.B. Baillière, Paris, 1829 (segunda edición); Prefacio de la primera edición 1828, reeditado por S. Douailler en el Corpus des oeuvres philosophiques de langue frangaise, Fayard, Paris, 1986, págs. 9-21.

(26) Victor Cousin, Fragments Philosophiques, A. Sautelet, Paris, 1826, pág. XLVII.

(27) Victor Cousin, Prefacio al Manuel d'histoire de la Philosophie de Tennemann, A. Sautelet, Paris, 1829, t. I, pág. XIII.

(28) Stendhal, Sketch of Parisian Sociely, 23/07/1828; ver Patrice Vermeren, "Une politique de l'institution philosophique, ou de la tactique parlementaire en matière de religion et de philosophie: Edgar Quinet et Victor Cousin", en: Corpus, N I, 1985, págs. 108-110.

(29) Victor Cousin, Cours d'Histoire de la Philosophie, Pichon et Didier, Paris, 1828; reedición Corprus des ouvres de philosophie de lungue fransaise, Fayard, Paris, 1991.

(30) E. A. Weisse, Über den gegenwärtigen Siandpunkt der philosophischen Wissenschaften in besonderer Beziehung auf das System Hegels, Leipzig, 1829, citado por Joachim Ritter, Hegel et la Révolution Fransaise, Beauchesne, Paris, 1970, pág. 16; Gwendoline Jarcyck y Pierre-Jean Labarrière, Le Syllogisme du ponuvoir: y-a-t-il une démocratie hégelienne?, Aubier, Paris, 1989.

(31) Victor Cousin, "Des principes de la révolution Française et du gouvernement représentatif", en: Revue des Deux-Mondes, 01/04/1851; ver también Victor Cousin, fustice et Charité, petits traités publiés par l'Academie des Sciences Morales et Politiques, Pagnerre et Paulin, Paris, 1848; y el segundo apéndice a Victor Cousin, Philosophie Ecossaise, Librairie Nouvelle, Paris, 1856 (tercera edición) págs. 342 y ss., en el cual Cousin elabora otra vez su leyenda de no haber dejado nunca de establecer y de sostener, en toda ocasión, la verdad y la belleza de la monarquía constitucional, no solamente bajo el gobierno de julio, sino también bajo los dos regímenes opuestos de la Restauración y de la República.

(32) Sobre las relaciones de Hegel y de Cousin, ver Franz Rosenzweig, Hegel et l'Etah, Puf, Paris, 1992, pág. 408 -traducción Gérad Bensoussan-; Martial Guéroult, Histoire de l'Histoire de la Philosophie, op. cit., t. III, págs. 716 y ss.

(33) Victor Cousin, Philosophie de Locke, Didier, Paris, 1861 (cuarta edición), pág. XIII. 
(34) Abbé Maret, Théosticêe chrétienne, ou comparaison de la notion chrétienne avec la notion rationaliste de Dieru, J. Leroux et Juby, Paris, 1850 (segunda edición), págs. 458-459.

(35) Pierre Leroux, "Du cours de philosophie de Schelling. Aperçu de la situation de la philosophie en Allemagne", en: Rerue Indépendunte, mayo, 1842, págs. 289-348.

(36) A. Véra, "M. Cousin et sa philosophie, a propos de ses Fragments et Souvenirs", 1865.

(37) "...a medida que la filosofia alemana se ha desarrollado más y que la hemos conocido mejor, nos hemos separado más abiertamente de ella, y se puede decir que la escuela que pretende hoy ser la heredera de M. Hegel no tiene adversarios más decididos que mis amigos y yo por la forma, por los principios, como por el método", escribe en 1866. Victor Cousin, Fragments de Philosophie Contemporaine, Didier et Durant, Paris, 1866 (quinta edición).

(38) Victor Cousin, Prefacio a los Fragments de Philosophie Cartésienne, Charpentier, Paris, 1845, pág. XI.

(39) Old Nol (seudónimo de Jules Barbey d'Aurevilly), "Quarante médaillons de l'Académie", en: Le Nain juune, setiembre/octubre, 1863.

(40) Victor Cousin, Prefacio a la segunda edición de Frugments Philosophiques, t. I, Ladrange, Paris, 1838 (tercera edición), pág. 32. 